Reprod. Nutr. Dévelop., 1984, 24 (6), 855-863.

\title{
Dispositif de collecte de l'effluent du rumen chez le Mouton.
}

\author{
J. COLLOMBIER $\left({ }^{* *}\right)$, C. DARDILLAT $(* *)$, J. SENAUD $\left({ }^{*}\right)$, C. A. GROLIËRE $\left({ }^{*}\right)$, \\ J.-P. JOUANY $\left(^{* *}\right)(1)$
}

(*) Laboratoire associé au C.N.R.S. 138, Biologie comparée des Protistes, Université de Clermont II, U.E.R. Sciences, Les Cézeaux, B.P. 45, 63170 Aubière, France.

${ }^{* *}$ ) Laboratoire de la Digestion des Ruminants, I.N.R.A., Theix, 63122 Ceyrat, France.

Summary. An apparatus for the collection of rumen effluent in sheep.

The apparatus, described in this paper, for collecting the rumen effluent (DCER) in sheep is composed of a flexible rubber probe which is introduced into the abomasum through a cannula $60 \mathrm{~mm}$ in diameter. The front end of the probe has a lateral hole which is pressed on the reticulo-omasal orifice (ORO) inside the omasum. A funnel-shaped collector is then introduced into the reticulum by a rumen cannula $75 \mathrm{~mm}$ in diameter ; the narrow part of the collector passes through the ORO and is applied to the sound hole in the omasum, its wide part lying on the internal ruminal side of the ORO. This apparatus does not appear to disturb the normal functioning of the ORO and permits the entire rumen effluent to be collected over periods of at least $3 \mathrm{hrs,} \mathrm{while} \mathrm{avoiding} \mathrm{mixing} \mathrm{the} \mathrm{effluent}$ with the contents of the omasum and abomasum.

\section{Introduction.}

La connaissance des phénomènes digestifs qui se déroulent dans chaque compartiment du tube digestif des Ruminants ne peut être acquise qu'en comparant qualitativement et quantitativement le flux entrant et le flux sortant de ce compartiment. En particulier, le rôle privilégié du réticulo-rumen dans la digestion de la ration ne peut être connu par les méthodes de bilan classique puisqu'il n'est pas possible de placer une fistule à la sortie du réseau, ou à l'entrée du feuillet, pour de simples raisons anatomiques. L'utilisation de marqueurs permet d'évaluer la quantité d'effluent sortant du rumen, mais n'autorise pas son analyse qualitative. Il est donc nécessaire, connaissant ce qui entre dans le rumen (ration alimen-

(1) A qui devront être adressées les demandes de tirés-à-part. 
taire), de récolter l'effluent à la sortie même du réseau, c'est-à-dire au niveau de l'orifice réticulo-omasal (ORO). Les méthodes utilisées jusqu'à présent ont consisté à essayer de collecter l'effluent du rumen sur l'animal vivant: Phillipson (1952) insère une canule en ébonite dans la partie pylorique de l'abosasum ; cette canule traverse la paroi abdominale et est reliée par un tube en verre à une canule du duodénum permettant ainsi une continuité du flux du liquide du rumen. Cet auteur signale que cette technique n'est pas satisfaisante. Weller et Pilgrim (1974) effectuent leurs prélèvements grâce à un tube introduit le plus près possible de l'orifice réticulo-omassal. Engelhardt et Hauffe (1975), Hauffe et Engelhardt (1975 a, b) utilisent, pour effectuer leur prélèvement, un manchon de nylon suturé sur l'orifice réticulo-omasal. Jouany (1978) prélève I'effluent du rumen à l'aide d'une canule du feuillet insérée directement dans le canal omasal. Toutes ces méthodes présentent plusieurs imperfections, dont les principales sont de ne pas récolter l'intégralité de l'effluent, et de ne pas éviter les reflux périodiques du contenu acide de la caillette qui vient se mêler à l'effluent du réseau dans le feuillet. Afin d'éviter ces inconvénients, nous avons mis au point, chez le Mouton, un dispositif qui capte l'effluent du rumen dans le réseau juste à l'avant de l'ORO, et le conduit jusqu'à un flacon collecteur placé à l'autre extrémité de la sonde, sous l'abdomen de l'animal, à travers une large fistule de la caillette.

\section{Matériel et méthodes.}

Description du dispositif de collecte de l'effluent du rumen (DCER) (fig. 1). L'appareil comporte plusieurs parties :

- une partie principale, ou sonde, tube de caoutchouc souple, de $30 \mathrm{~mm}$ de diamètre et de 25 à $30 \mathrm{~cm}$ de longueur ; sa paroi est armée d'un fil d'acier hélicoïdal adhésil (Silastic type $A$; SERAP) afin d'éliminer tout risque de réaction inflammatoire des tissus en contact : une ouverture (œil) latérale de $30 \mathrm{~mm}$ de diamètre est pratiquée un peu en arrière de l'extrémité proximale aveugle; cette sonde est introduite, par son extrémité proximale, par une canule de la caillette, placée sous l'abdomen du Mouton dans la partie inférieure de la grande courbure (Collombier, 1981); elle franchit l'orifice omaso-abomasal situé à la verticale de la canule, traverse le feuillet, et son œil latéral est appliqué contre l'ORO (fig. 2). - Un collecteur en entonnoir, constitué par un anneau de $75 \mathrm{~mm}$ de diamètre, entouré de silicone (Dispersion CAF4 ; Isolants du Rhône) et relié par un manchon de latex très fin à une bague métallique de $30 \mathrm{~mm}$ de diamètre; ce collecteur est introduit dans le réseau par la fistule du rumen ; puis, à travers l'ORO, la bague métallique est ajustée à l'œil latéral de la sonde, par simple rotation, à l'aide d'un mandrin ; ainsi, l'anneau s'applique contre l'ORO, du côté du réseau, et seul le manchon de latex est en contact avec la paroi de l'ORO.

- Un dispositif de fixation de l'extrémité distale de la sonde à la canule de la caillette, comportant une plaque (L) solidaire de la sonde, se fixe à la rondelle (C) ajustée sur la canule par un système de ressorts $(E)$, de tiges filetées $(G)$ et d'écrous (M). 
A Canule de la caillette.

A

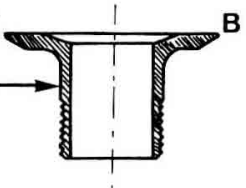

B Ecrou de la canule

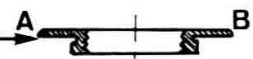

\section{Support supérieur de} la sonde

Coupe

D Connecteur de l'oeil de la son'de au réseau -

E Ressort extensible.

F Chapeau de la sonde

G Tige filetée

H Entretoise flexible, armée, enrobée de silicone

Qutil servant à fixer $D$ dans $F$

I Vanne à3orifices de réintroduction de l'effluent dans la caillette

J Joint d'ètanchéité

$K$ Robinet de la vanne
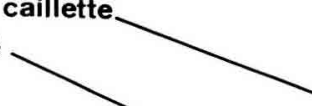

B.

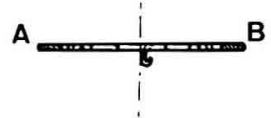

L Support inférieur de la sonde

M Ecrou de serrage
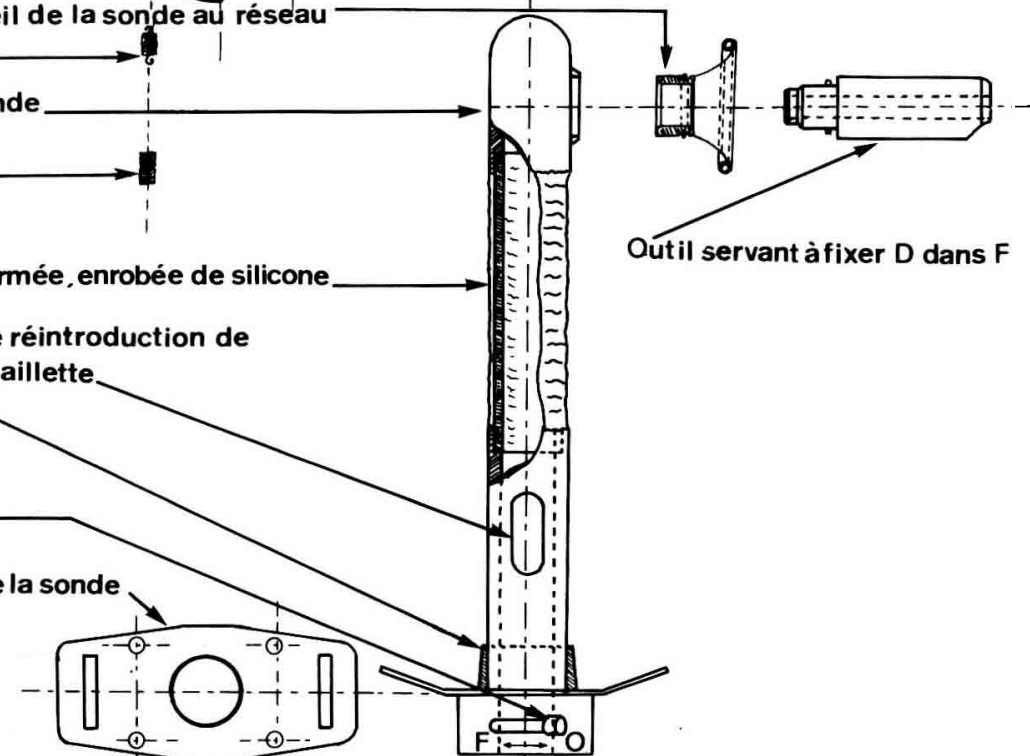

N Bouchon disperseur de l'effluent dans la caillette

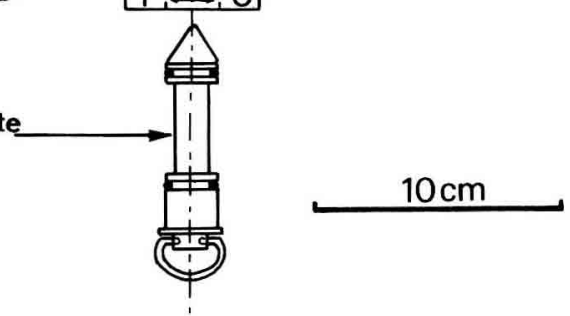

FIG. 1. - Schéma de l'appareil de collecte de l'effluent du rumen (DCER).

- Une vanne à 3 orifices (I), commandée par un robinet $(K)$ et un bouchon disperseur (N), autorise l'écoulement direct de l'effluent du rumen dans la caillette en dehors des périodes de prélèvement.

L'ensemble, une fois posé et fixé, est maintenu en place à l'aide d'un harnais placé autour de l'abdomen de l'animal ; ce harnais porte le flacon collecteur dans lequel s'écoule l'effluent du rumen. 


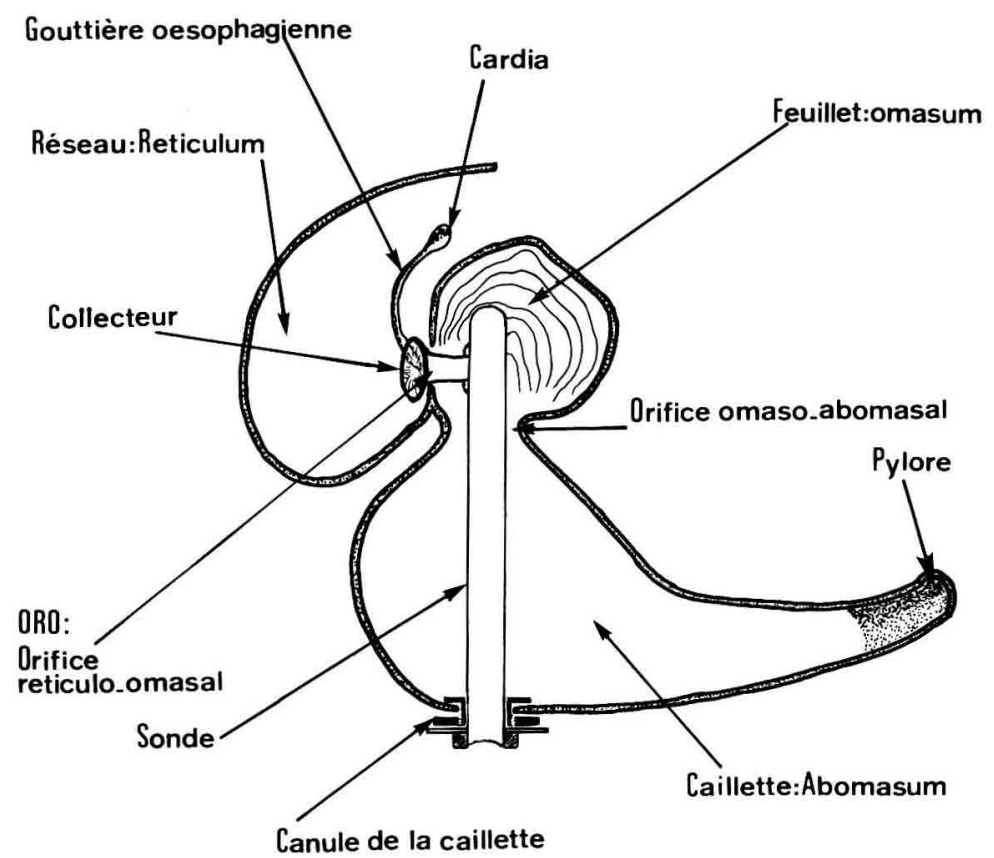

FIG. 2. - Position de la sonde et du collecteur par rapport aux compartiments stomacaux du Mouton.

Animaux. - Sept moutons âgés de plus de 3 ans, de race Texel, castrés, pesant environ $75 \mathrm{~kg}$, ont été munis d'une canule du rumen (diamètre : $75 \mathrm{~mm}$ ) et d'une canule de la caillette (diamètre : $60 \mathrm{~mm}$ ). La fistulation de la caillette (Collombier, 1981), calquée sur celle du rumen, ayant conduit, chez 4 moutons, à une mauvaise étanchéité et à l'extroversion de la caillette, il a été nécessaire d'utiliser, pour les 3 autres animaux, un tissu chirurgical de synthèse, le Rhodergon (RhônePoulenc), déjà utilisé dans les zones à forte acidité lors de l'implantation de canules duodénales effectuées par fistulation par voie transthoracique extra-pleurale chez le porc (Laplace et Tomassone, 1970), qui a permis une colonisation cellulaire autour de la canule. Seuls ces derniers animaux ont été utilisés pour les études de transit de contenu à la sortie du rumen.

Aliments. - Afin d'éviter l'obstruction du DCER par de trop grosses particules sortant du rumen, les animaux ont reçu une ration à base de pellets de luzerne déshydratée (62\%), additionnée de lactosérum doux en poudre $(13 \%)$, de paille traitée à la soude (NIS, COFNA) (25\%) broyée et agglomérée, et complémentée en minéraux et en vitamines. Les animaux ont ingéré chaque jour $1470 \mathrm{~g}$ de matière sèche d'aliment et $15 \mathrm{~g}$ du mélange minéral et vitaminique, répartis en 2 repas $(8 \mathrm{~h} 30$ et $17 \mathrm{~h})$. Pendant les collectes, l'aliment est retiré aux animaux, s'il en reste une certaine quantité. 
Prélèvements. - L'effluent du rumen a été collecté grâce au DCER, soit complet, soit sans collecteur avec seulement l'œil latéral de la sonde appliqué contre l'ORO ; après avoir éliminé les premières salves qui suivent l'installation de la sonde, nous avons recueilli l'effluent évacué dans un bécher attaché sous le ventre de l'animal, toutes les demi-heures. Afin d'éviter de trop longues durées de manipulation et de récolte de l'effluent, qui risquaient de perturber les animaux, nous avons effectué des collectes pendant trois périodes distinctes, mais complémentaires, qui ont permis de recouvrir l'intervalle de temps $(T)$ entre le repas du matin $(8 \mathrm{~h} 30)$ et celui du soir ( $17 \mathrm{~h}$ ), à savoir : de $8 \mathrm{~h} 30$ (avant le repas) à 11 h 30 ; de 11 h 30 à 14 h 30 ; de 14 h 30 à 17 h (avant le repas). Ces collectes sur l'intervalle $(T)$ ont été répétées 6 fois sur chacun des 3 moutons utilisés.

A des fins de comparaison, du contenu de rumen a été prélevé à des intervalles réguliers de $1 / 2$ ou $1 \mathrm{~h}$, par la canule du rumen, pendant les mêmes périodes.

Mesures. - Le pH a été déterminé sur le contenu du rumen et sur l'effluent de manière extemporanée et ponctuelle durant toute la période de cette collecte. - Evaluation du transit réticulo-omasal : une solution à $60 \mathrm{~g} / \mathrm{l}$ de polyéthylèneglycol (PEG) avec un débit de $45 \mathrm{ml} / \mathrm{h}$ a été infusée pendant plusieurs jours avant les prélèvements; $48 \mathrm{~h}$ après le début de l'infusion et toutes les heures (de $8 \mathrm{~h} 30$ à $17 \mathrm{~h}$ ) du contenu de rumen a été prélevé et les échantillons ont été cumulés pour en mesurer la concentration moyenne en PEG (Hyden, 1955) pendant I'intervalle de temps $T$. Dès que cette concentration a atteint une valeur plateau, la collecte d'effluent du rumen par le DCER a été entreprise, en recueillant les volumes évacués toutes les $1 / 2 \mathrm{~h}$, et les échantillons ont été cumulés pour obtenir, pour l'intervalle de temps $T$, d'une part le volume total évacué, d'autre part la concentration moyenne en PEG de l'effluent. Simultanément, nous avons prélevé du contenu de rumen et déterminé sa concentration moyenne en PEG. On a pu ainsi calculer le débit moyen théorique de la phase liquide à la sortie du rumen à partir de la concentration en PEG de l'effluent $\left(\mathrm{Dr}_{2}\right)$, et à partir de la concentration en PEG du jus de rumen $\left(\mathrm{Dr}_{3}\right)$, et le comparer au débit réel mesuré à partir du volume d'effluent recueilli grâce au DCER $\left(D_{1}\right)$.

\section{Résultats.}

Transit réticulo-omasal de l'effluent du rumen (tabl. 1).

Le volume de la phase liquide de l'effluent récolté par le DCER pendant le temps $\mathrm{T}$ est sensiblement identique à celui qui peut être calculé en déterminant la concentration en PEG de l'effluent et en connaissant la quantité de PEG infusée pendant la même période.

En revanche, le débit moyen théorique de la phase liquide à la sortie du rumen, déterminé le jour des collectes à l'aide de la concentration en PEG du jus de rumen $\left(D R_{3} b\right)$, est toujours inférieur au débit horaire mesuré directement avec le DCER $\left(D r_{1}\right)$. En comparant $\mathrm{Dr}_{2}$ (débit théorique calculé par la concentration en PEG de l'effluent) avec $\mathrm{Dr}_{3} \mathrm{~b}$, on constate que le PEG est plus dilué dans l'effluent que dans la phase liquide du contenu du rumen, ce qui indique que la teneur en 
TABLEAU 1

Débit de la phase liquide de l'effluent du rumen $\left({ }^{*} \mathrm{~m} / / \mathrm{h}\right)$.

Périodes de prélèvement

8 h 30 à 11 h $30 \quad 11$ h 30 à 14 h $30 \quad 14$ h 30 à 17 h $\quad$ T $(8$ h 30 à 17 h)

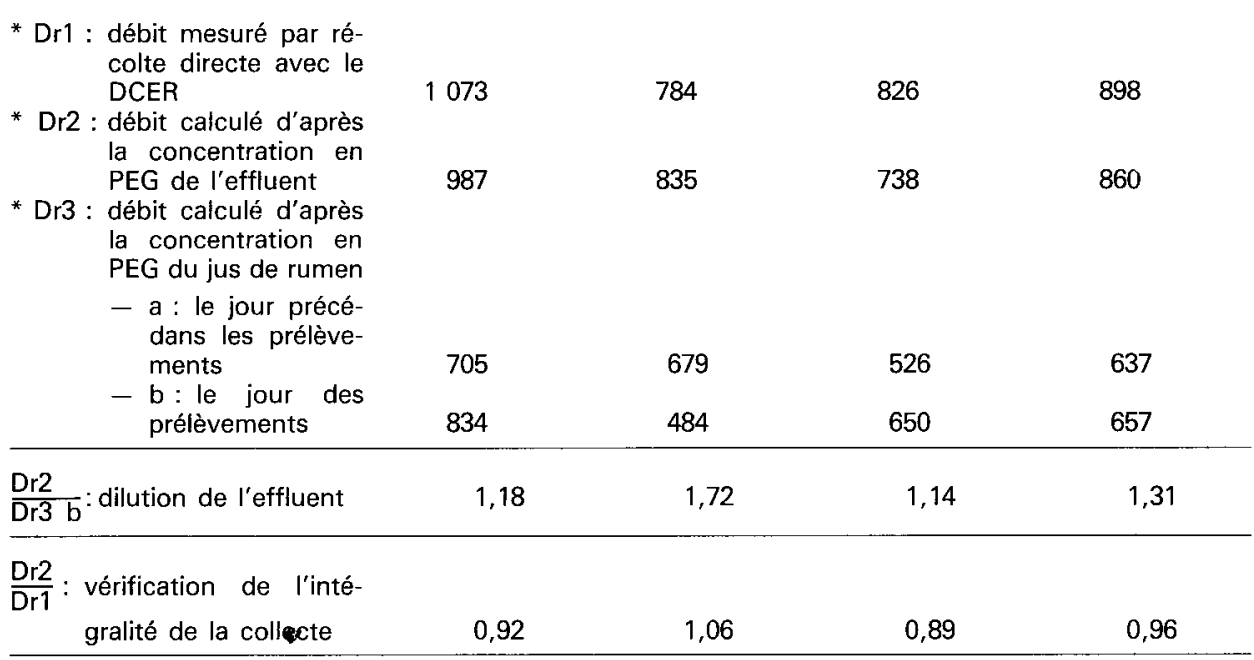

matière sèche de l'effluent est plus faible que celle du rumen ; cette dilution de l'effluent est égale au rapport de la concentration en PEG dans le rumen sur celle de l'effluent, ou au rapport des débits $\frac{\mathrm{Dr}_{2}}{\mathrm{Dr}_{3} \mathrm{~b}}$ pour chaque période de mesure.

En calculant le débit moyen à la sortie du rumen par dosage du PEG dans le contenu du rumen le jour qui précède la pose du DCER $\left(\operatorname{Dr}_{3} a\right)$ pendant $T$, on obtient des valeurs de débit moyen tout à fait comparables à celles obtenues le jour des collectes de l'effluent $\left(\mathrm{Dr}_{3} \mathrm{~b}\right)$; ceci indique que la pose du DCER n'a pas d'effet sensible sur le débit de l'effluent du rumen et sur le fonctionnement du sphincter de I'ORO.

Le rapport $\frac{D r_{2}}{\mathrm{Dr}_{1}}$ est proche de 1 pour l'ensemble de la période $T$, ce qui prouve que le DCER récolte pratiquement l'intégralité de l'effluent : en effet, si on considère que le débit $\mathrm{Dr}_{2}$, à la sortie du rumen, mesuré par la méthode de dilution du PEG, est le débit de référence correct, et puisque le débit mesuré à la sortie du DCER $\left(D r_{1}\right)$ est égal à ce débit de référence $\mathrm{Dr}_{2}$, on peut considérer que notre technique est satisfaisante.

Le $p H$ de l'effluent est toujours supérieur à celui du contenu du rumen au même moment (tabl. 2) ; la différence entre les deux semble augmenter à mesure que l'on s'séloigne du repas du matin et que les valeurs absolues s'élèvent. 
TABLEAU 2

Comparaison des $\mathrm{pH}$ de l'effluent et du liquide du rumen dans différentes conditions de prélèvements.

\begin{tabular}{lcc}
\hline & Rumen & Effluent \\
\hline $\mathrm{pH}$ moyen (17 mesures) entre $8 \mathrm{~h} 30$ et $17 \mathrm{~h}$ & $6,30 \pm 0,2$ & $7,00 \pm 0,25$ \\
$\mathrm{pH}$ moyen (4 mesures) entre $8 \mathrm{~h} 30$ et $10 \mathrm{~h} 30$ & $6,51 \pm 0,11$ & $6,95 \pm 0,10$ \\
$\mathrm{pH}$ moyen entre $10 \mathrm{~h} 30$ et $14 \mathrm{~h} 30$ & & \\
- sur 8 prélèvements ponctuels & $6,21 \pm 0,20$ & $7,05 \pm 0,20$ \\
- Sur 10 prélèvements ponctuels & $6,64 \pm 0,11$ & $7,40 \pm 0,15$ \\
- sur 10 autres prélèvements ponctuels & $6,70 \pm 0,10$ & $7,40 \pm 0,10$ \\
\hline
\end{tabular}

\section{Discussion.}

La méthode de récolte de l'effluent du rumen que nous avons employée est originale, bien qu'elle soit dérivée de la technique décrite par Engelhardt et Hauffe (1975).

L'efficacité du DCER peut être jugée d'après deux critères : le maintien en fonctionnement normal de l'ORO, la quantité d'effluent recueilie par rapport à celle qui sort effectivement du rumen :

Le transit du contenu digestif du réseau au feuillet est en relation directe avec l'ouverture de l'ORO, mais il est aussi influencé par la position des lèvres de la gouttière œsophagienne, et par le gradient de pression existant entre les deux compartiments (Bueno, 1975) ; si, habituellement, la présence d'un corps étranger dans la zone réflexogène de l'ORO gêne son mécanisme, il est évident que la suture d'un manchon de nylon sur l'ORO, comme l'ont pratiquée Engelhardt et Hauffe (1975) au niveau de l'orifice omaso-abomasal, doit perturber fortement les contractions de l'ORO. Avec le DCER, le contact du seul manchon de latex très souple avec la paroi de l'ORO n'est pas traumatisant ; en effet, nous avons vérifié que la sortie d'effluent par le DCER s'effectue par salves de $30 \mathrm{ml}$ environ séparées par des intervalles de $2 \mathrm{~min}$, ce qui correspond aux données physiologiques normales chez un animal sain (Bueno, 1975 ; Poncet et al., 1982) ; le débit normal réticulo-omasal n'est pas multiplié par 2 ou 3 par la pose du DCER dans l'ORO, ce qui est le cas si on $y$ introduit un tube rigide. Enfin, nous avons pu vérifier en introduisant un doigt dans I'ORO muni du DCER que la fermeture du sphincter est tout à fait normale. Cependant, afin d'éviter divers troubles éventuels, le dispositif n'a pas été laissé en place pendant plus de $9 \mathrm{~h}$. Une fois mis en place dans l'animal, le DCER est utilisé au moment de chaque collecte. Son utilisation temporaire se révèle satisfaisante pour une récolte intégrale de l'effluent du rumen $\left(\mathrm{Dr}_{1} \# 1\right)$.

$$
\overline{\mathrm{Dr}_{2}}
$$

II reste toutefois des perfectionnements à apporter à l'appareil (mode d'introduction et de fixation, amélioration de la vanne à trois voies) pour pouvoir réaliser des collectes sur une période continue de $24 \mathrm{~h}$. II faut aussi améliorer le système d'arrimage du collecteur à la sonde, car cette opération est délicate. 
- Les caractéristiques de l'effluent recueilli avec le DCER sont différentes de celles du contenu du rumen. Le PEG est plus dilué dans l'effluent que dans le rumen : il est donc probable que la méthode classique de détermination du débit de la phase liquide à la sortie du rumen, à partir de la concentration en PEG dans le liquide du rumen, sous-estime la valeur réelle de la vidange du rumen ; la dilution de l'effluent $\mathrm{Dr}_{2}>\mathrm{Dr}_{3} \mathrm{~b}$ pourrait être due à l'eau de boisson conduite directement à l'ORO par la gouttière œsophagienne sans se mélanger au contenu du rumen (Weller et Pilgrim, 1974), et plus probablement à la salive qui arrive régulièrement dans le réseau. En outre, le $\mathrm{pH}$ de l'effluent du rumen est plus élevé que celui du contenu du rumen, ce qui avait déjà été observé par Weller et Pilgrim (1974). Ce phénomène peut s'expliquer également par l'apport d'eau ou de salive ( $\mathrm{pH}$ de la salive $=8,4$ à 8,7), ou/et par l'absorption importante des acides gras volatils dans la zone de l'ORO (Weller et Pilgrim, 1974).

Puisque notre dispositif recueille l'effluent au niveau de l'ORO, nous n'avons jamais observé de chutes du $\mathrm{pH}$ dues à des reflux périodiques du contenu de la caillette vers le feuillet, comme cela avait été montré par d'autres auteurs (Weller et Pilgrim, 1974 ; Wyburn, 1980).

\section{Conclusion}

Le dispositif de collecte de l'effluent du rumen que nous proposons peut donc être considéré comme une amélioration sensible des procédés employés auparavant, puisqu'il ne perturbe pas le transit digestif, qu'il récolte l'intégralité de l'effluent, tout en évitant que celui-ci ne se contamine avec le contenu des autres compartiments de l'estomac du Ruminant.

Reçu en février 1984, Accepté en juin 1984.

Remerciements. - Nous tenons à remercier MM. P. Thivend et J. Grain pour les discussions et les conseils apportés dans la réalisation de l'appareil et dans la rédaction du manuscrit.

\section{Références}

BUENO L., 1975. Les fonctions motrices et digestives du feuillet. Th. Doct. ès Sci. nat., Univ. Toulouse, $n^{\circ} 652,206 \mathrm{p}$.

COLlOMBIER J., 1981. Contribution à l'étude du rôle des Protozoaires Ciliés du rumen dans l'apport d'azote microbien dans le duodénum du Ruminant. Th. Doct.-Ing., Univ. de Clermont II, Série DI, $\mathrm{n}^{\circ} 105,95 \mathrm{p}$.

ENGELHARDT W. v., HAUFFE R., 1975. Role of the osmasum in absorption and secretion of water and electrolytes in sheep and goats, 216-230. In Mc. DONALD I. W., WARNER A. C. I., "Digestion and metabolism in the Ruminant", ed. The Univ. New England Publ. Unit, Australia. 
HAUFFE R., ENGELHARDT W. v., 1975 a. Funktionen des Blättermagens bei kleinen Hauswiederkäuern. I. Zufluss und Verweildauer von festen Teilchen und von Flussigkeit. Zbl. Vet. Med., A, 22, 149-163.

HAUFFE R., ENGELHARDT W. v., 1975 b. Funktionen des Blättermagens bei kleinen Hauswiederkäuern. III. Resorption von Wasser. Zbl. Vet. Med., 1, 22, 283-295.

HYDEN S., 1955. A turbidimetric method for the determination of higher polyethylène glycols in biological materials. K. Lautbrhögsk. Ann., 22, 139-145.

JOUANY J. P., 1978. Contribution à l'étude des Protozoaires Ciliés du rumen: leur dynamique, leur rôle dans la digestion et leur intérêt pour le ruminant. Th. Doct., Univ. Clermont II, $\mathrm{n}^{\circ} \mathrm{d}^{\prime}$ ordre 256, 2 volumes, $195 \mathrm{p}$.

LAPLACE J. P., TOMASSONE R., 1970. Evacuation gastro-duodénale chez le Porc. Fistulation chronique par voie thoracique extrapleurale; recherche d'une étude mathématique de l'évaluation. Ann. Zootech., 19, 303-332.

PHILLIPSON A. T., 1952. The passage of digesta from the abomassum of sheep. J. Physiol., London, 116, 84-97.

PONCET C., YVAN M., LÉVEILLE M., 1982. Electromagnetic measurements of osmodenal digesta flow in canulated sheep. Reprod. Nutr. Dévelop., 22, 651-660.

WELLER R. A., PILGRIM A. F., 1974. Passage of protozoa and volatile fatty acids from the rumen of the sheep and from a continuous in vitro fermentation system. Br. J. Nutr., 32, 341-351.

WYBURN R. S., 1980. The mixing and propulsion of the stomach contents of ruminants, 35-51. In RUCKEBUSCH Y., THIVEND P., Digestive physiology and metabolism in ruminants. M.T.P. Press Ltd., Lancaster, England. 